\title{
Methods for improving models for condensed phase equilibrium calculations
}

\author{
A. Marcilla, M.M. Olaya, M.D. Serrano, J.A. Reyes-Labarta* \\ Chemical Engineering Department, University of Alicante, Apdo. 99, Alicante 03080, Spain
}

\section{A R T I C L E I N F O}

\section{Article history:}

Received 10 August 2009

Received in revised form 18 November 2009

Accepted 21 December 2009

Available online xxx

\section{Keywords:}

Phase equilibria

Correlation

NRTL

Local composition models

LLE

LLSE

\begin{abstract}
A B S T R A C T
Local composition models like UNIQUAC, NRTL and the UNIFAC group contribution method are used the world over in the synthesis and design of separation processes, as well as for a large number of other applications of industrial interest. They can qualitatively describe the equilibrium behaviour of systems of different complexities but, sometimes, not with the precision required for the design of separation equipment. Regrettably, this precision is not even achieved for the LLE of many conventional type 1 and some type 2 ternary systems. High deviations in correlation results are not rare, thus showing that more capable and flexible models are needed. The analysis of the topology of the Gibbs energy of mixing function, and therefore the knowledge of the geometrical conditions that this function has to fulfil, is a valuable tool that has allowed avoiding inconsistencies in the use of the existing methods, to ascertain their capabilities and limitations to reproduce complex systems and additionally to suggest new models that improve the flexibility and accuracy needed. In this work, a simple equation based on the NRTL model, that takes into account such topological conditions, is proposed and its capabilities are tested with selected systems.
\end{abstract}

(C) 2009 Elsevier B.V. All rights reserved.

\section{Introduction}

The synthesis and design of separation processes requires the use of models to represent the phase equilibrium data, which involve the previous correlation of experimental data to obtain the values of the corresponding parameters. The possibility of determining the number of existing phases for certain conditions with a unique set of parameters would be interesting to avoid serious problems in many industrial processes such as distillation or pumping [1]. Despite the fact that simultaneous correlation of all the existing equilibrium regions is very advantageous for design calculations, correlation parameter sets are frequently found in literature that do not describe all the existing regions [2-7], or that describe more immiscibility regions than actually exist (i.e. p. 71 and 436 in DECHEMA Chemistry Data Series [8]). The capability of describing the complete equilibrium behaviour of the experimental system is a measurement of the correlation success and the flexibility of the model.

Existing software packages like DECHEMA Data Preparation Package [9] and the regression utilities of Chemical Engineering simulators like ChemCAD [10] offer the possibility of experimental data correlation using different classical models. They have been widely used and their capabilities, with respect to equilibrium data correlation, are well-known. Nevertheless, they have been

\footnotetext{
* Corresponding author. Tel.: +34 965 903789; fax: +34 965903826

E-mail address: ja.reyes@ua.es (J.A. Reyes-Labarta).
}

designed for the simplest LLE behaviour (types 1 and 2) and do not allow the correlation of type 0 LLE, type 3 LLLE or type 4 LLSE systems (Treybal classification [11]).

However, these complex behaviours are not so infrequent and it would be desirable to correlate them for certain industrial applications. Type 0 isle ternary systems commonly result from the simultaneous presence of a weak acid and a weak base, that reversibly form a soluble salt, which has a lower miscibility than either the free acid or the free base, leading to the liquid-liquid phase separation [12], or they also occur in ternary systems of two polymers and a low molecular weight solvent [13]. Systems exhibiting a LLLE region (type 3 ) are also of considerable interest for industrial processes involving surfactants that find application in the production of cosmetics, herbicides, paints, lubricants, etc. [14]. They are especially important in the petroleum engineering industry where surfactants are used to recover residual oil [15-17]. And finally, type 4 ternary systems (LLSE) are of interest mainly due to the salting-out effect used in many industrial applications such as extractive fermentation $[18,19]$, removal of contaminants from industrial effluents [5], extractive crystallization [20], etc.

Correlation data always require comparison between the experimental and the calculated equilibrium data. The accuracy of the results obtained depends on many factors which are related to the quality of the experimental data and how are they used, the model selected, as well as to the calculation algorithms and the capability to overcome the convergence problems related to them. The optimization method used must determine the best set of parameters for the model that minimizes the objective function of the 
deviations among the experimental and calculated compositions (i.e. O.F. $\left.(x)=\sum_{s=1}^{n} \sum_{i=1}^{c} \sum_{k=1}^{p}\left(x_{i, s}^{\exp }-x_{i, s}^{\mathrm{cal}}\right)_{k}^{2}\right)$ for the $c$-components present in the $p$ phases of the $n$ tie-lines. Nevertheless, the calculated compositions must be real equilibrium compositions (i.e. fulfilling the isoactivity criterion: for a two phases system O.F. $(a)=$ $\left.\sum_{s=1}^{n} \sum_{i=1}^{c}\left(a_{i, s}^{\mathrm{I}}-a_{i, s}^{\mathrm{II}}\right)^{2}=0\right)$. Problems may arise while searching adequate parameters if the set of experimental data are not uniformly distributed in the equilibrium space. In this case it would be advisable to prepare a set of "experimental" data obtained by interpolating among the actually obtained, and use this new set of homogeneously distributed data, to compare with the calculated data.

Existing equilibrium calculation strategies (for instance the procedure used in the Liquid-Liquid Equilibrium Data Collection [8]) give good results in most cases, but they may lead to uncertainties and problems in others, as evidenced by errors found in regression reports of programs and some published results, even for type 1 systems, where O.F. $(a) \neq 0$ [21]. There are many questions and problems to be solved with respect to correlation of equilibrium data and many research efforts are being devoted to solve them.

In previous papers [22-26] robust calculation methods, based on the topological analysis of the Gibbs energy of mixing function $\left(g^{\mathrm{M}}=\left(\Delta_{\text {mix }} G\right) / R T\right)$, were developed. The topological analysis of $g^{\mathrm{M}}$ is a valuable tool that allows deducing the geometrical conditions that the $\mathrm{g}^{\mathrm{M}}$ function has to fulfil, in order to reproduce certain equilibrium behaviour in the whole composition diagram, and clearly evidences any existing calculation inconsistency. The topological flexibility of the $g^{\mathrm{M}}$ function defined by each model determines its capability to reproduce different equilibrium behaviours.

The previously developed methods [22-26] resulted in a robust computer program that has allowed the determination of the capabilities and limitations of the NRTL equation [27] for correlating equilibrium data between condensed phases in ternary systems. NRTL equation was selected as representative of thermodynamic local composition models, and is one of the most frequently used model. Complex behaviours of type 0 (island), 1, 2 (LLE), 3 (LLLE) and 4 (LSE, LLSE, LShE, LLShE and LSShE) ternary systems, comparing NRTL [27] with electrolytic-NRTL [28-30] in the last case, were analyzed in detail. The studied systems had not been previously correlated if they were, results were inconsistent with the number of miscible pairs in the system. From the results obtained, it was concluded that NRTL can qualitatively describe the behaviour of the studied complex systems, but provided very high deviations (up to $6 \%$ ), which was not useful for engineering purposes.

Frequently, correlation deficiencies of equilibrium data are associated with the dependence of the results on the initial guesses for the variables to be calculated, as well as to convergence calculation problems due to the non-linear equilibrium conditions, and other reasons. However, the topological analysis of the $g^{\mathrm{M}}$ functions presented in this paper shows that, in many cases, the intrinsic limitations of the classical models are the very reason of making it impossible to find a solution of the problem. In the present work, some NRTL model limitations are illustrated and the necessity of more flexible models is discussed. Moreover, a possible way to develop such a model overcoming the limitations is presented. Thus, a new equation based on a simple modification of the NRTL model is proposed and its extended capability is illustrated with some correlations.

\section{Limitations of NRTL model}

The NRTL model is based on binary interaction parameters, so LLE data for an $n$-component system can be calculated from the values of the two binary parameters corresponding to the $n$ binary subsystems included in the multicomponent system if the non-
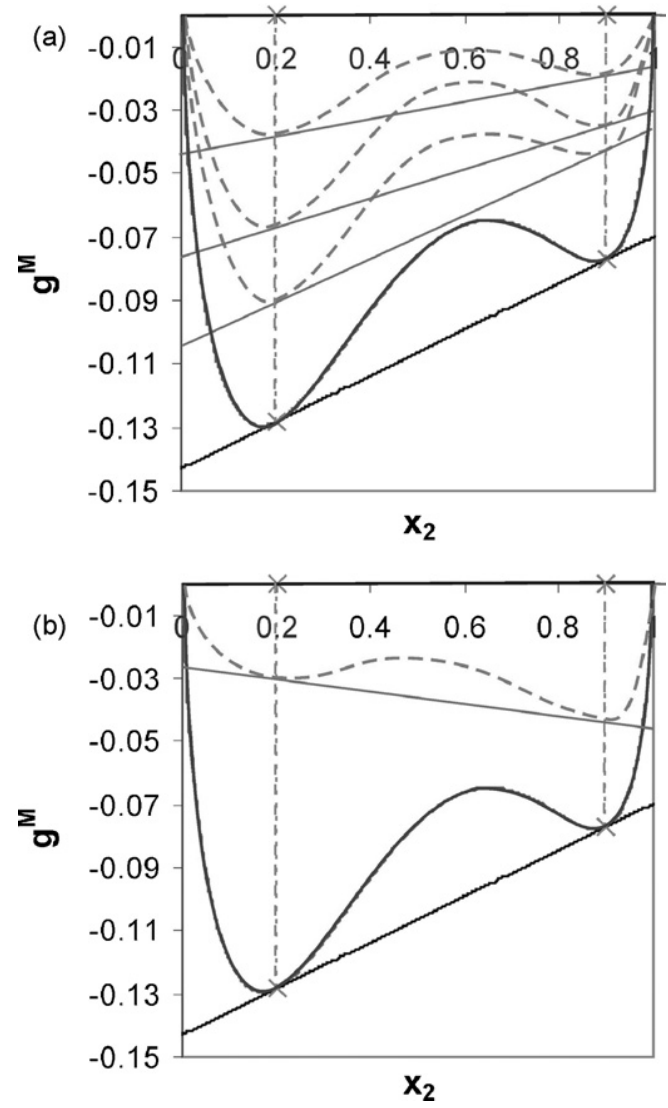

Fig. 1. Oneness of heterogeneous $g^{\mathrm{M}}$ binary curves with NRTL (-) for a given equilibrium $\left(A_{12}(\mathrm{~K})=257.92, A_{21}=632.90, \alpha=0.2 T=25^{\circ} \mathrm{C}\right)$. Discontinuous lines $(----)$ represent qualitative curves that cannot be generated with NRTL: (a) different values for the minima, and (b) different slope of the tangent line.

randomness factor is considered constant, as it usually is.

The relation of the $g^{\mathrm{M}}$ with the equilibrium compositions, as provided by a model, is through the tangent plane condition [31,32] (the derivatives of the $g^{\mathrm{M}}$, related to the activities) but not through the value of the $\mathrm{g}^{\mathrm{M}}$. Obviously, any model produces a unique curve $g^{\mathrm{M}}$ vs. composition once the corresponding parameters are fixed. This is also true for local composition models. And if the model has two parameters only two conditions could be specified for such $g^{\mathrm{M}}$ vs. composition functions. For instance, if the compositions of the two phases in equilibrium are fixed there is only a single curve, if any, that the NRTL equation with the corresponding pair of parameters (unique pair of $A_{i j}$ for $\alpha$ constant) could predict being compatible with such behaviour. No more sets of $A_{i j}$ parameters can be found that reproduce different curves with a common tangent line at the same equilibrium compositions.

Fig. 1a shows the $g^{\mathrm{M}}$ vs. composition function predicted by NRTL when fixing the equilibrium composition at $x_{2}^{\mathrm{I}}=0.2$ and $x_{2}^{\mathrm{II}}=0.9$. The solid line represents the unique curve that NRTL predicts (for $\alpha=0.2$ ). The discontinuous curves represent possible behaviours that cannot be reproduced by the NRTL model. Such $g^{\mathrm{M}}$ vs. composition curves may be necessary for representing different ternary systems having a binary with the specified compositions (i.e. at $x_{2}^{\mathrm{I}}=0.2$ and $x_{2}^{\mathrm{II}}=0.9$ as in the previous case) in equilibrium and tie-lines in the ternary region with different slopes. Fig. 1b shows $g^{\mathrm{M}}$ vs. composition curves providing common tangent lines with slope of contrary sign to that predicted by NRTL for $x_{2}^{\mathrm{I}}=0.2$ and $x_{2}^{\mathrm{II}}=0.9$. This type of behaviour cannot be predicted by the NRTL equation, which is again a serious limitation when trying to correlate different ternary systems with a binary having the same mutual solubility and ternary tie-lines with different slopes. 

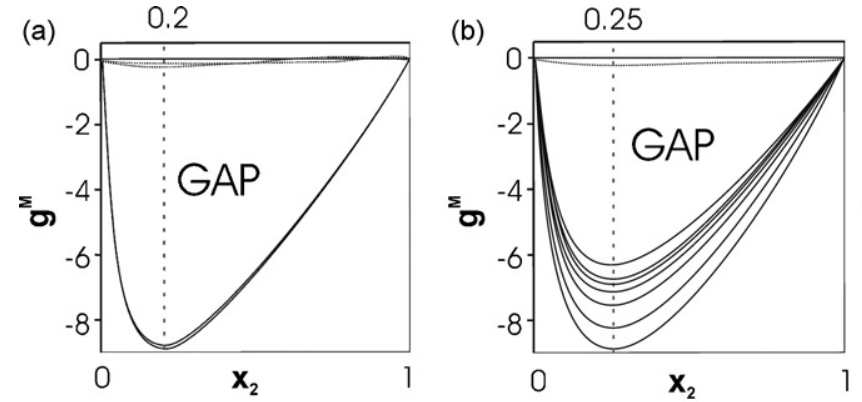

(d) 0.35

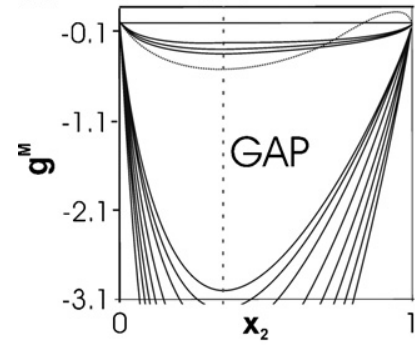

(e)

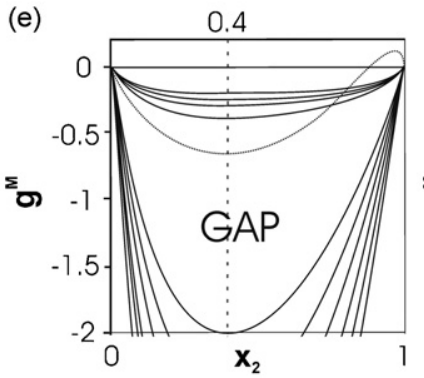

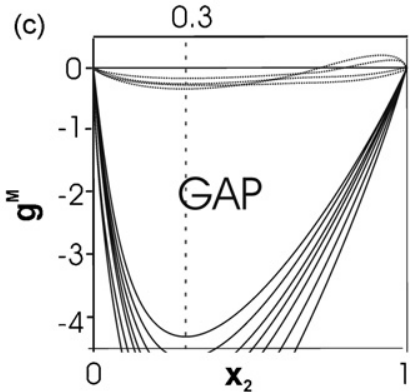

(c)

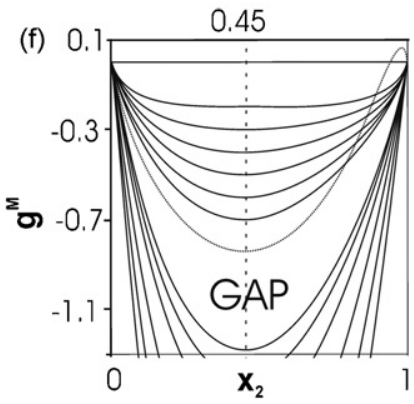

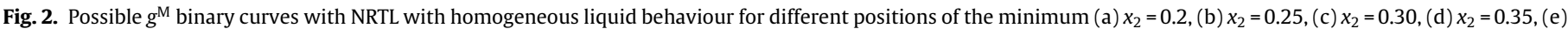
$x_{2}=0.4$ and (f) $x_{2}=0.45 . g^{\mathrm{M}}$ curves with phase splitting are represented by dotted lines.

We have run a systematic study on the capability of NRTL to reproduce homogeneous $g^{\mathrm{M}}$ vs. composition binary curves that may clarify the reasons of the limitations of this model to fit some LLE data, and the difficulty found in some cases in the convergence of the optimization methods. A wide range of $x_{i, \min }(0.20-0.45)$ and $g_{\min }^{\mathrm{M}}$ values (between -0.2 and -9 ) were evaluated and the NRTL binary parameters reproducing each minimum were found [33]. Some of the situations unavoidably led to $2 \mathrm{~L}$ phase splitting of the binary system, i.e., to obtain a minimum of the given $g^{\mathrm{M}}$ value at the given composition could only be predicted if the curve presents another minimum or a situation where phase splitting occurred. In most of the cases, no curve was found with the $x_{2, \min }$ and $g_{\min }^{\mathrm{M}}$ values specified. As a consequence, gaps were found where solutions for homogeneous binary behaviour did not exist. It was observed that when $x_{2, \min }$ moves away from 0.5 (increasing or decreasing), the gap increases. Fig. 2 illustrates this behaviour for six positions of $x_{i, \text { min }}$ below 0.5 . Scale is different since it has been selected in each case to focus on the gap. It must be considered that these equations are symmetric, thus yielding similar curves for $x_{i, \min }$ corresponding compositions in the $0.5-1$ interval.

The existence of such gaps reveals situations where it is not possible to find NRTL $-g^{\mathrm{M}}$ curves compatible with homogeneous behaviour of the system. This limitation together with the oneness of the curve reproducing partial miscibility for a binary system restricts the capability of the NRTL model to correlate different systems and can also explain many convergence problems during correlation procedures.

\section{Examples of the consequences of the limitations on the data correlation}

New applications of the existing models have already been investigated and the correlation methods improved to try to obtain the optimum results that these models can provide. Nevertheless, the nature is very complex and the phase equilibrium behaviour for many systems cannot be described with the precision required for the design of the separation equipment. Regrettably, this precision is not even achieved for the LLE of some conventional type
1 ternary systems. It seems that existing models are not capable of providing adequate results for many systems, and it is not difficult to find in the literature clear examples of such incapacity. It seems clear that there is a necessity of developing new models capable of representing such type of systems with the required accuracy. The study of the topology of the $g^{\mathrm{M}}$ function is an indispensable tool to achieve this goal, as we will illustrate in the following examples.

We have selected two examples to illustrate the limitations of NRTL and the reasons for such limitations. The first one is the type 1 system methanol(1) + diphenylamine (2) + cyclohexane (3) at $298 \mathrm{~K}$ (DECHEMA, vol. 2, p. 129). In that data collection, this system is correlated with the NRTL model giving a set of parameters that predict tie-line slopes contrary to the experimental ones and we tried to improve the correlation with NRTL with no results.

$3 \mathrm{D} \mathrm{g}^{\mathrm{M}}$ surface is shown in Fig. 3a, where the tangent plane comprising a tie-line calculated with the model (i.e. for a given composition value of $x_{2}=0.0136$ ) is also included. A magnification of the LLE region is necessary to appreciate the curvature of the partially miscible binary 1-3 (Fig. 3b). As we have commented in the previous section, the partially miscible binary system can only be reproduced by a single curve with a defined curvature. The best set of parameters found for the homogeneous binary subsystems 1-2 and $2-3$, in combination with the partially miscible binary pair $1-3$, are responsible for the contrary slopes of the calculated tie-lines. It is clearly observed in Fig. 3b that the tangent plane containing the tie-line, marks two shadowed areas in the surroundings of the equilibrium phases where the plane is very near the surface. In this figure the calculated tie-line and the projection of the experimental tie-line with molar fraction of component 2 in right phase closest to $x_{2}=0.0136$ are shown. It is clearly observed that the slopes of the two tie-lines are contrary. Fig. 4 shows the complete composition diagram in the ternary composition triangle together with the $g^{\mathrm{M}}$ binary curves.

The second selected system is the type 3 system 1-hexanol (1) + nitromethane (2) + water (3) at $294 \mathrm{~K}$ (DECHEMA, vol. 2, p. 69). In the data collection, this system is not correlated with the NRTL model. In a previous work [26] the binary tie-lines (LLE) and the tietriangle (LLLE) were simultaneously correlated with NRTL showing 
(a)
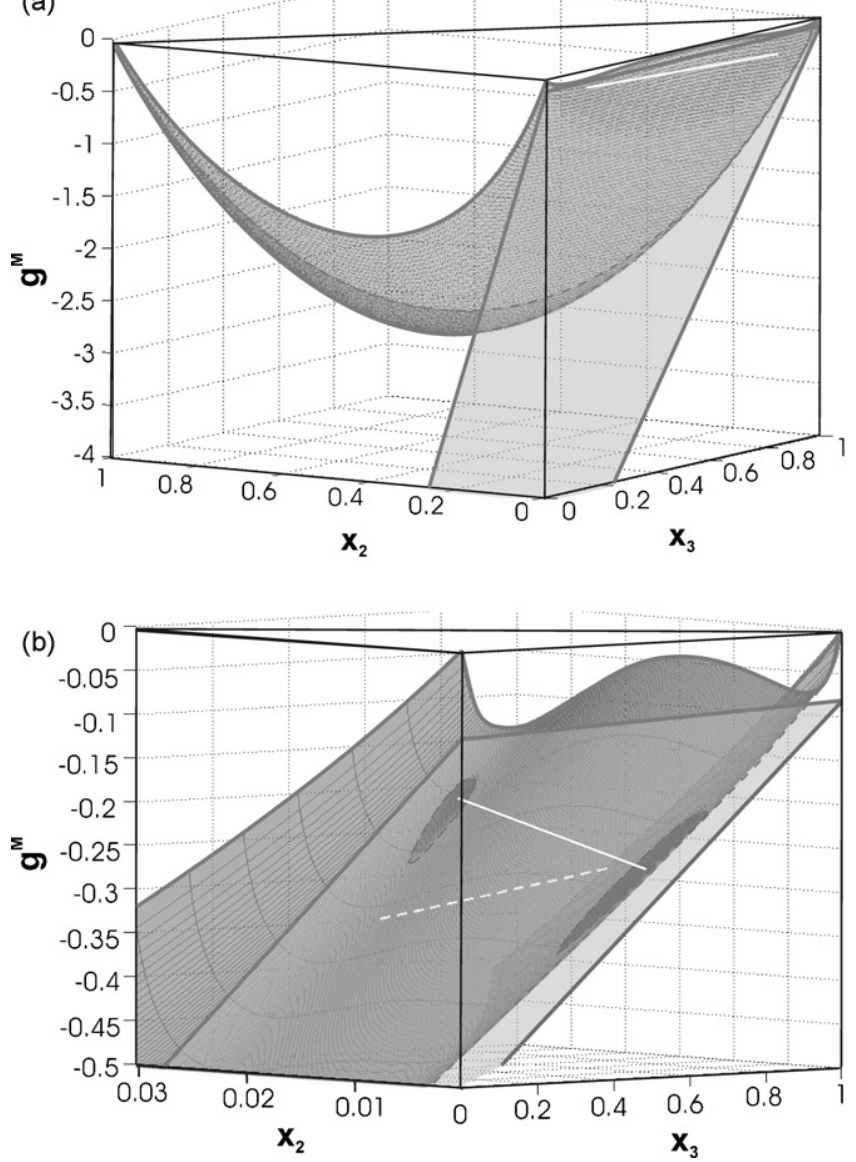

Fig. 3. Gibbs energy surfaces for NRTL model (parameters in Table 1) with tangent plane comprising a tangent line (continuous line) of a given composition value of $x_{2}=0.0136$ ): (a) global view and (b) magnification of the LLE region where the projection on the plane of the experimental tie-line that is nearest $x_{2}=0.0136$ is also represented for comparison (discontinuous line). that the very narrow LLLE tie-triangle and the LLE data for the 1-2 binary subsystem could not be simultaneously correlated with enough accuracy, though the behaviour could be represented qualitatively. Fig. 5 shows the complete composition diagram together with the $g^{\mathrm{M}}$ binary curves obtained with NRTL.

The corresponding 3D $g^{\mathrm{M}}$ surface is shown in Fig. 6, where the tangent plane comprising the tie-triangle calculated with the model is also included.

These examples clearly show that the lack of flexibility of the models (i.e.: NRTL as representative of all others) to represent different behaviours of the $g^{\mathrm{M}}$ binaries, and correspondingly the ternary surfaces, is the cause of the lack of ability to correlate certain experimental systems, including the apparently simplest ones as the type 1 systems.

\section{What characteristics should be expected for a model?}

Obviously, any new model should be based on binary parameters, be symmetrical with respect to the components and fulfil the characteristics of the existing models. But from the previous considerations, as well as for other widely accepted reasons, a model for phase equilibrium calculations should have a set of characteristics and capabilities:

(a) The first and more important one is that the model should have enough flexibility to produce different $g^{\mathrm{M}}$ binary curves compatible with the same mutual solubility, thus enabling the possibility of representing different ternary systems with the same binaries.

(b) Secondly, and related to the first, the model should avoid, as much as possible, the existence of the gaps in the $g^{\mathrm{M}}$ curves previously discussed.

(c) It would be desirable that the new model should be a modification of a classical model, including it in its formulation. In that case the parameters already obtained for the classical model would normally be the best initial guesses for the new model.

(d) The model should have the minimum number of parameters, but enough so as to assure the required flexibility.

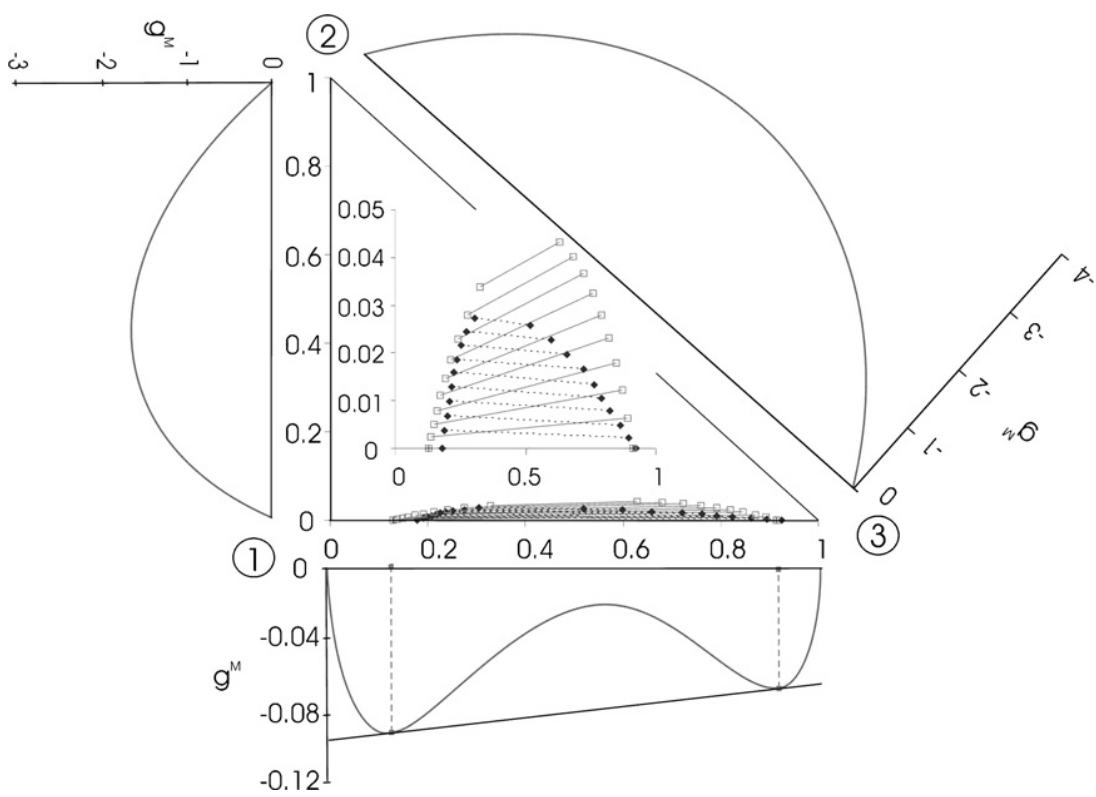

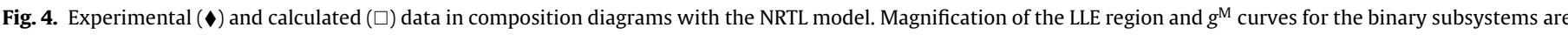
included. Parameters in Table 1. 


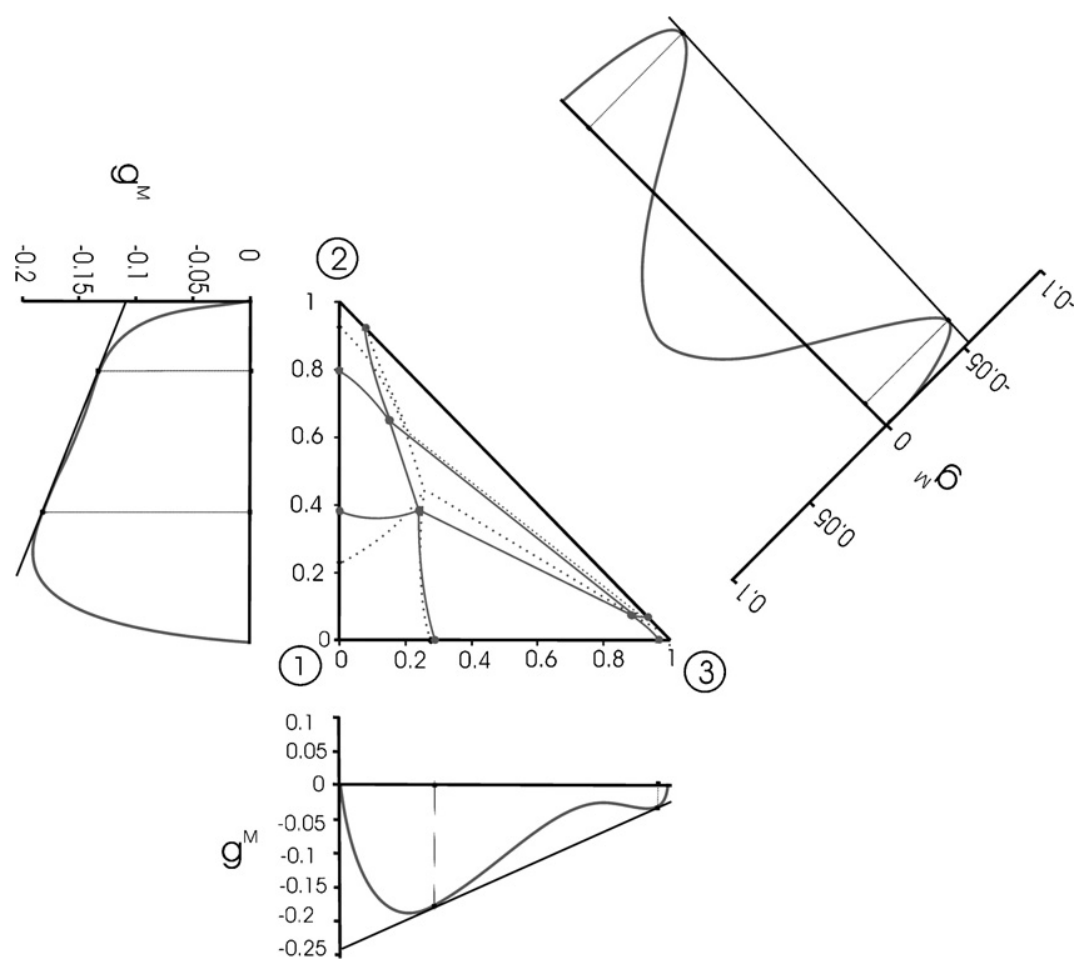

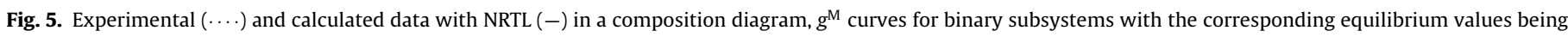
included. Parameters in Table 2.

\section{An example of a new model improving the results}

There is a much extended thought that a model should have no more than two (three in special cases) parameters per binary pair. A classical topic is that a model with more parameters is supposed to provide better results, although they are strongly questioned and very few examples can be found in literature. Nevertheless, from our point of view, none of these two topics are founded. Regarding the first one, it is impossible to provide a mathematical equation expressing the dependence of the $g^{\mathrm{M}}$ with the required flexibility with only two parameters. From this it is evident that more parameters are needed. Regarding the second one, the use of more parameters is not necessarily a guarantee of improving the results. The mathematical expression should provide the required flexibility and yield $g^{\mathrm{M}}$ curves compatible with the behaviour of the real systems. Also the expression of $g^{\mathrm{M}}$ should avoid the possibility of false solutions.

Taking these considerations into account we have tested many modifications of the existing local composition models (i.e. considering a dependency of the parameters of NRTL with composition, different relative contributions between ideal and excess Gibbs energies, etc.) and empirical equations, such as Gaussian based expressions, which have not succeeded in improving the fitting of data of equilibrium between condensed phases. From our experience in the topological analysis of $g^{\mathrm{M}}$ surfaces, it can be concluded that many empirical models for the excess Gibbs energy, with a promising capability to reproduce equilibrium phase splitting for binary systems, easily predicted multiple meta-stable or unstable regions in the ternary equilibrium diagram. Furthermore, certain types of systems required the use of a higher number of parameters in order to extend the possibility of reproducing the existing equilibrium regions or specific behaviour for the tie-line slopes, which obviously reduced the utility of the model.
According to the abilities searched, a modification based on the local composition NRTL model is suggested that should be considered only as an example. Other models could be developed, but this is very simple and solves many of the requirements listed above. The expression for the Gibbs energy of excess is:

$\frac{G^{E}}{R T}=\sum_{i} F_{i} x_{i} \frac{\sum_{j} \tau_{j i} G_{j i} x_{j}}{\sum_{l} G_{l i} x_{l}}$

where $F_{i}$ are the new parameters proposed for the modified model. The rest of the parameters are defined the same as in the original model [27]. It includes a new parameter per component.

This model allows the filling in of the gaps found for NRTL as represented with discontinuous lines in Fig. 7. Furthermore, the model can provide sets of curves with different slopes for the common tangent line to the $g^{\mathrm{M}}$ vs. composition curves at the same specified equilibrium compositions, and even slopes of contrary sign. Fig. 8 shows those possibilities for some selected cases.

The proposed model allows a greater flexibility which will be tested by fitting the selected two previously selected systems, already correlated with NRTL in order to compare the results obtained.

For both systems, the optimization of the parameters ( $F_{i}$ and $\left.A_{i j}\right)$ has been carried out by using the simplex flexible method, with the objective function defined as:

O.F. $(x)=\min \sum_{s=1}^{n} \sum_{i=1}^{c} \sum_{k=1}^{P}\left(x_{i, s}^{\exp }-x_{i, s}^{\mathrm{cal}}\right)_{k}^{2}$

where exp and cal, respectively, refer to experimental and calculated compositions for the $c$-components present in the $p$ phases of the $n$ tie-lines.

All tie-lines fulfil the common tangent plane [31,32] criterion, that it is preferred to the isoactivity condition because it 


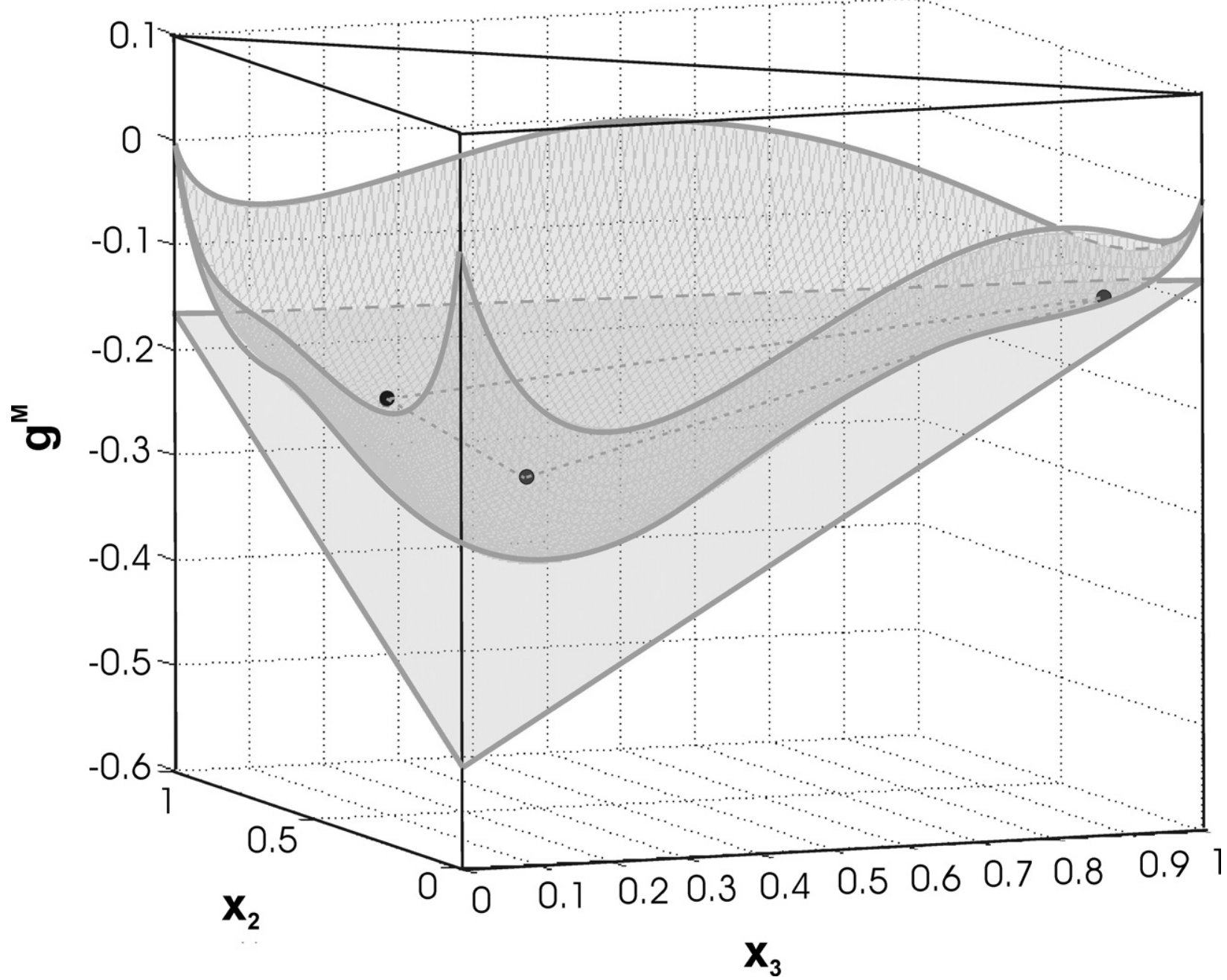

Fig. 6. Gibbs energy surface for the NRTL model (parameters in Table 2) with the tangent plane comprising the tie-triangle. The 3L phases are also represented in the figure $(\bullet)$
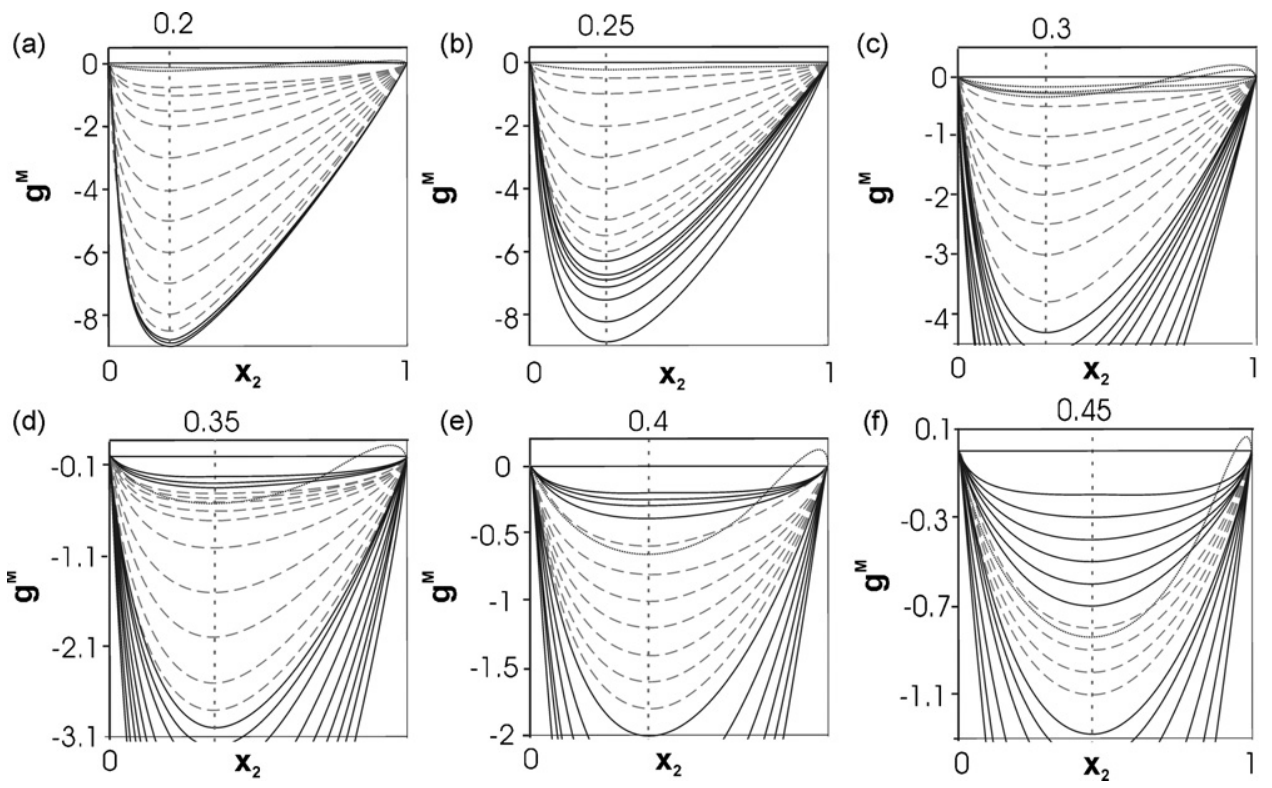

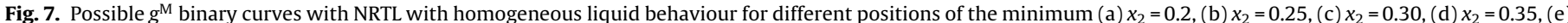
$x_{2}=0.4$ and (f) $x_{2}=0.45$. Gaps filled by the modified model are represented by discontinuous lines and $g^{\mathrm{M}}$ curves with phase splitting are represented by dotted lines. 


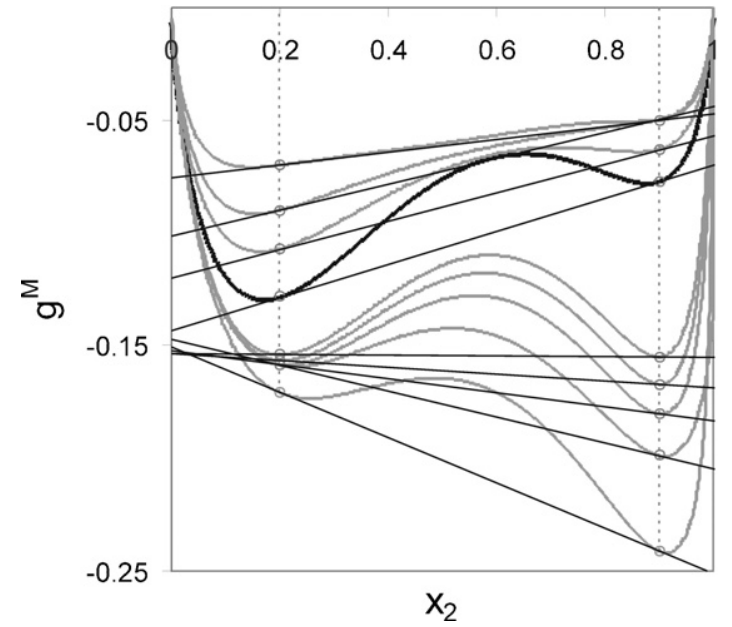

Fig. 8. Different $g^{\mathrm{M}}$ binary curves provided by the new model to represent the same equilibrium compositions $\left(x_{2}^{\mathrm{I}}=0.2, x_{2}^{\mathrm{II}}=0.9\right)$. Different values for the minima and different slopes of the tangent line are possible.

describes the equilibrium more precisely, avoids false solutions and accelerates convergence [34]. The corresponding set of nonlinear equations, formulated according to Iglesias-Silva et al. [35], are solved with the Newton-Raphson method.

Parameters and deviations are collected in Tables 1 and 2. Standard deviation is calculated as in the DECHEMA database [8]:

$\sigma=100 \sqrt{\frac{\sum_{s=1}^{n} \sum_{i=1}^{c} \sum_{k=1}^{p}\left[\left(x_{i, s}^{\mathrm{exp}}-x_{i, s}^{\mathrm{cal}}\right)_{k}^{2}\right]}{2 c n}}$

\subsection{Application of the new model to the type 1 system}

The previously considered type 1 system has been correlated again with the proposed model (Eq. (1)). Since it has been concluded that the slopes of the ternary calculated tie-lines depend on the
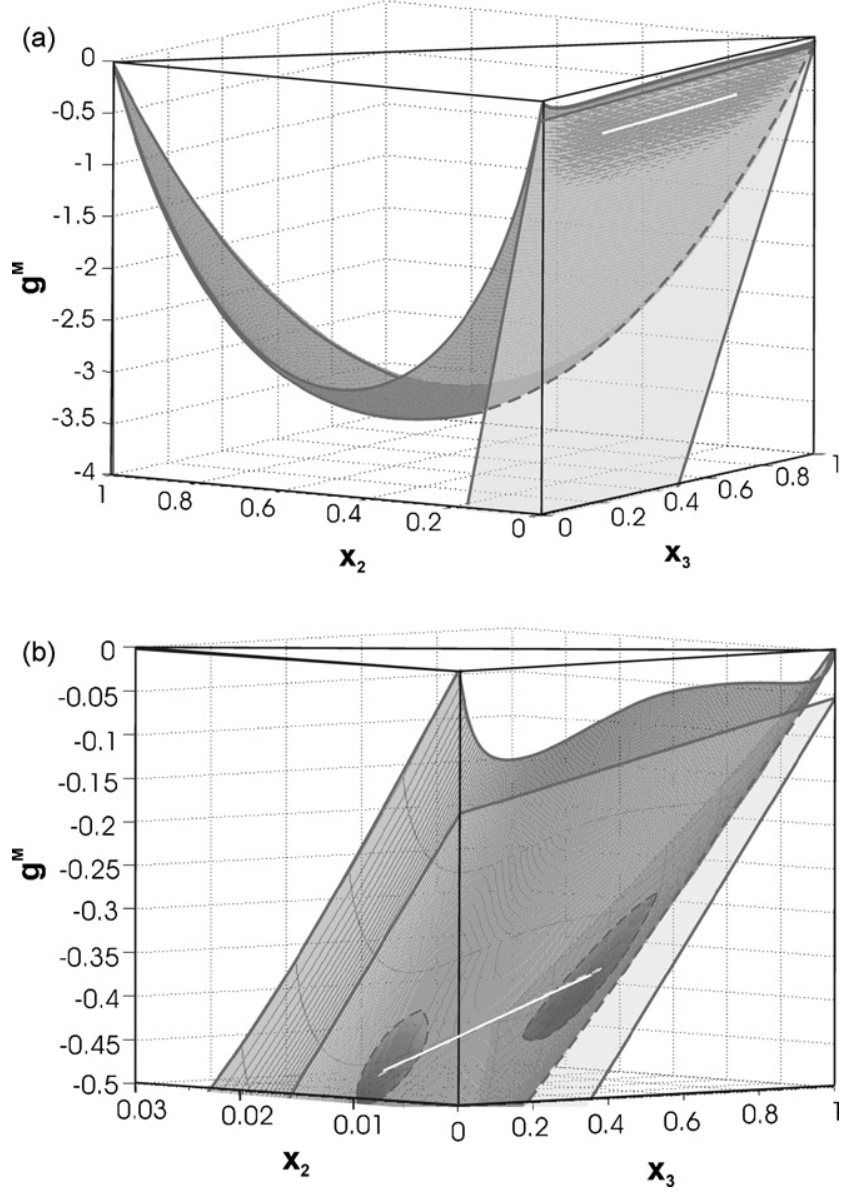

Fig. 10. Gibbs energy surfaces for the new model (parameters in Table 1) with tangent plane comprising a tangent line (continuous line) of a given composition value of $x_{2}=0.0136$ ): (a) global view and (b) magnification of the LLE region where the projection on the plane of the experimental tie-line that is nearest $x_{2}=0.0136$ is also represented for comparison (discontinuous line).
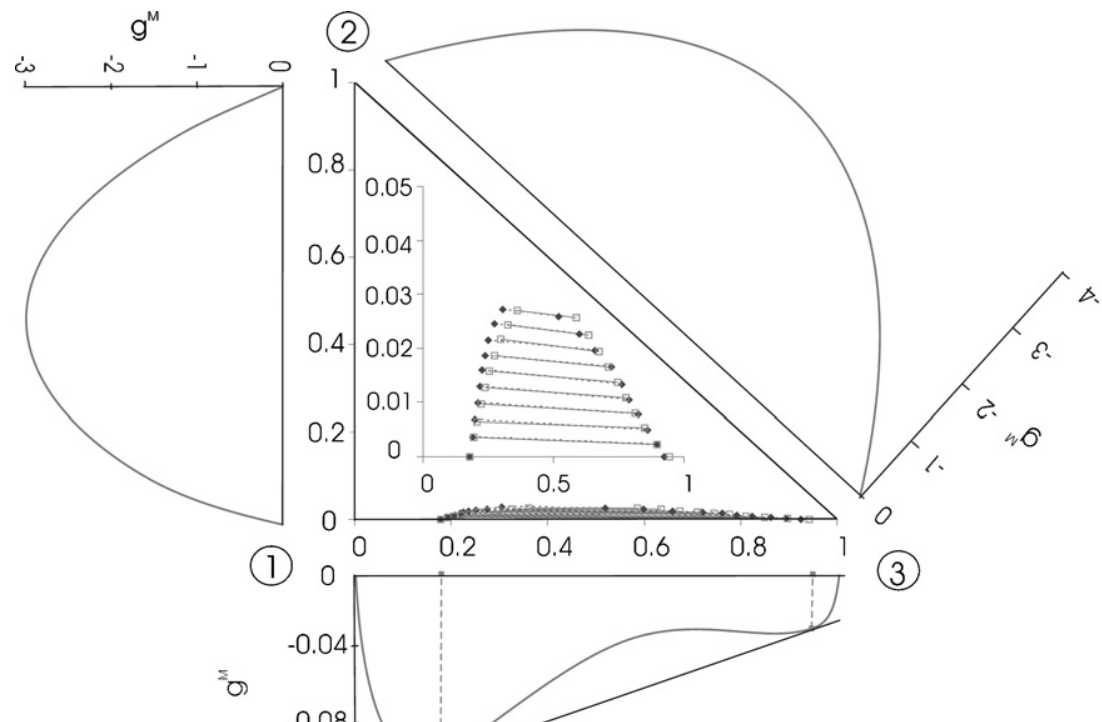

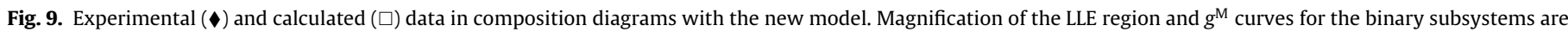
included. Parameters in Table 1.

Please cite this article in press as: A. Marcilla, et al., Methods for improving models for condensed phase equilibrium calculations, Fluid Phase Equilibr. (2010), doi:10.1016/j.fluid.2009.12.026 
Table 1

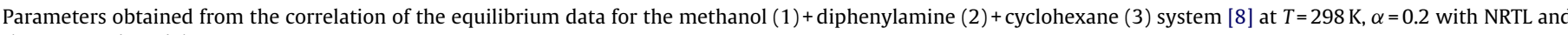
the proposed model.

\begin{tabular}{|c|c|c|c|c|c|c|c|c|}
\hline & & \multicolumn{3}{|c|}{$A_{i j}($ NRTL model [8]) } & \multicolumn{3}{|c|}{$A_{i j}($ new model) } & \multirow[t]{3}{*}{$F_{i}$} \\
\hline & & \multicolumn{3}{|l|}{$j$} & \multicolumn{3}{|l|}{$j$} & \\
\hline & & 1 & 2 & 3 & 1 & 2 & 3 & \\
\hline \multirow{4}{*}{$i$} & 1 & - & 873.57 & 379.39 & - & -731.45 & 50.984 & 0.174 \\
\hline & 2 & -1245 & - & -987.32 & -3112.6 & - & -193.02 & 1.915 \\
\hline & 3 & 578.07 & -856.11 & - & 3203.15 & -170.13 & - & 13.082 \\
\hline & & & $\sigma=4.08$ & & & $\sigma=2.47$ & & \\
\hline
\end{tabular}

Table 2

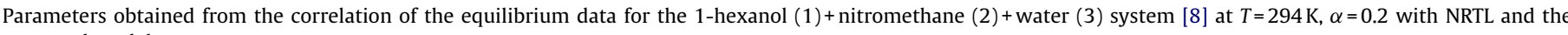
proposed model.

\begin{tabular}{|c|c|c|c|c|c|c|c|c|}
\hline & & \multicolumn{3}{|c|}{$A_{i j}($ NRTL model [8]) } & \multicolumn{3}{|c|}{$A_{i j}($ new model $)$} & \multirow[t]{3}{*}{$F_{i}$} \\
\hline & & \multicolumn{3}{|l|}{$j$} & \multicolumn{3}{|l|}{$j$} & \\
\hline & & 1 & 2 & 3 & 1 & 2 & 3 & \\
\hline \multirow{4}{*}{$i$} & 1 & - & 163.46 & -2.3341 & - & 15.130 & -561.09 & 1.473 \\
\hline & 2 & 577.60 & - & 492.78 & 560.89 & - & 744.63 & 1.068 \\
\hline & 3 & 1060.9 & 564.33 & - & 1333.69 & 646.86 & - & 0.634 \\
\hline & & & $\sigma=5.93$ & & & $\sigma=1.57$ & & \\
\hline
\end{tabular}

relative values of the $g^{\mathrm{M}}$ binary curves, initial values for the $F_{i}$ and $A_{i j}$ parameters were proposed after studying the effect of changes in $g^{\mathrm{M}}$ binary curves representations.

Results obtained are shown in Fig. 9. It can be observed that the modified model considerably improves the data correlation: tie-lines are coherent since they have the same slopes as the experimental ones and are very close to them, providing a much lower deviation than NRTL.
Fig. 10 shows $g^{\mathrm{M}}$ surface for this system obtained by the correlation with the new model. In this figure the tangent plane containing a tie-line calculated with the new model (for a given composition value of $x_{2}=0.0136$ ) is included. Differences between the 3D surfaces can be observed by comparing Figs. 3a and 10a, where for instance, the homogeneous binary $\mathrm{g}^{\mathrm{M}}$ curves for subsystems 1-2 and 2-3 are quite different. Again, a magnification of the LLE region is necessary to observe the curvature of the partially miscible binary

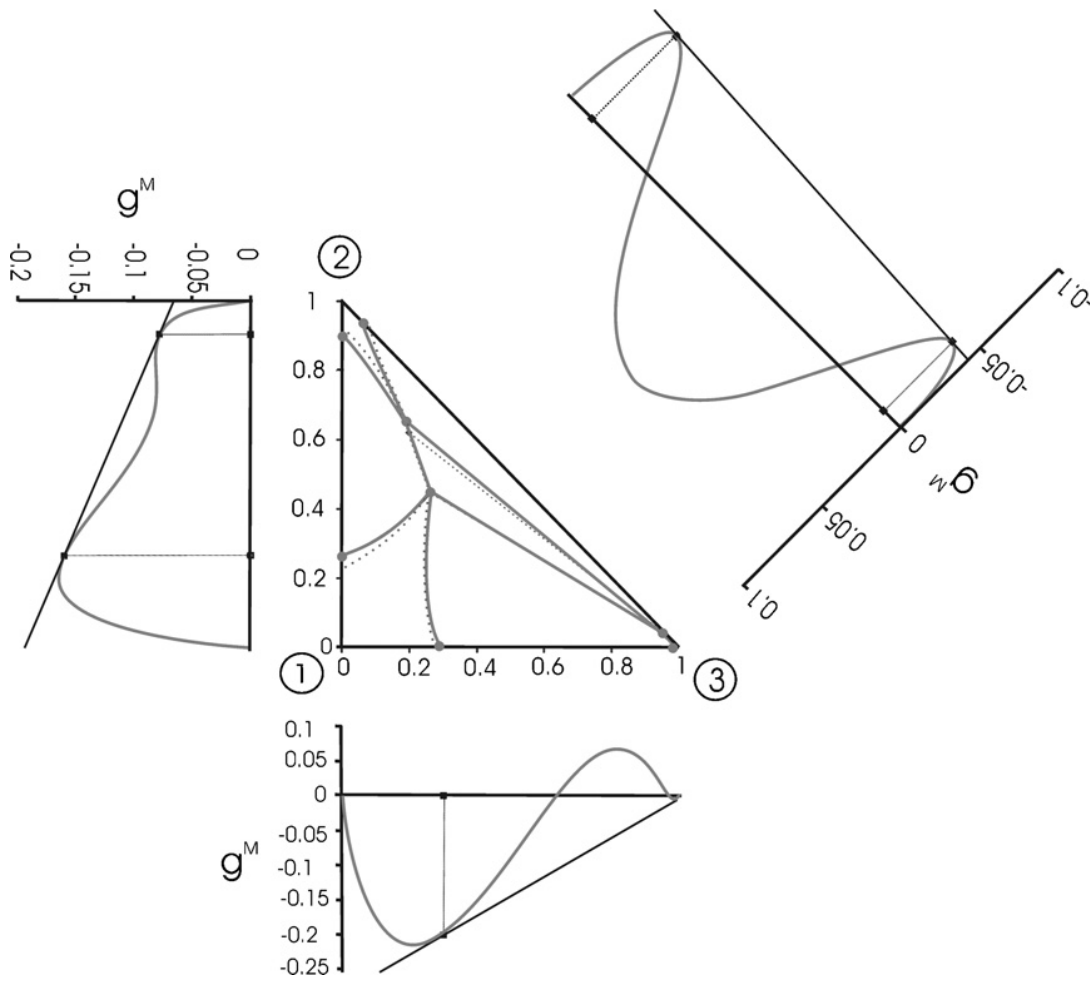

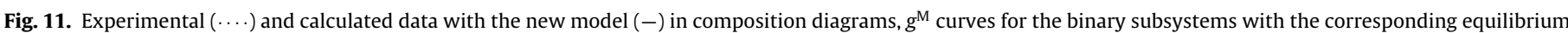
values are included. Parameters in Table 2. 


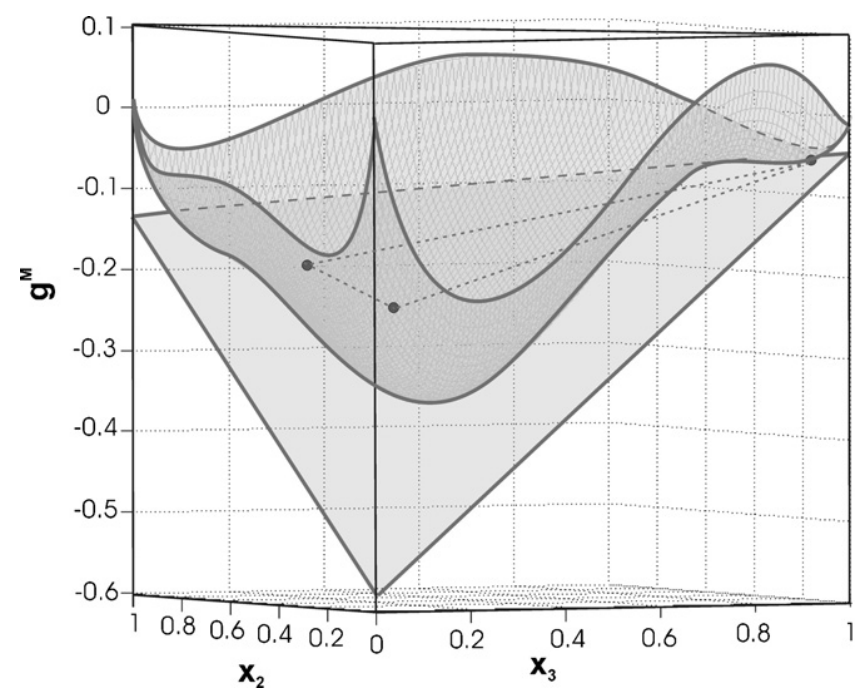

Fig. 12. Gibbs energy surface for the new model (parameters in Table 2) with the tangent plane comprising the tie-triangle. The 3L phases are also represented in the figure (•).

1-3 system (Fig. 10b). The projection of the experimental tie-line with molar fraction of component 2 closest to $x_{2}=0.0136$, on the previous tangent plane, is also shown (Fig. 10b). It can be seen that both lines are now almost coincident.

From Figs. 9 and 10, it can be observed that the $g^{\mathrm{M}}$ curves for 1-2 and 2-3 binaries with a homogeneous liquid behaviour are more similar with the modified model, which causes a decrease in the tie-line slopes. Furthermore, the $g^{\mathrm{M}}$ curve for the partially miscible binary $1-3$ is considerably different since the tangent line to the $g^{\mathrm{M}}$ curve has a higher slope and the curvature is less pronounced. NRTL could not predict this curve with any set of parameters. All these characteristics allow for predicting a smaller binodal curve closer to the experimental one with calculated tie-lines very similar to experimental data. The modifications of the $\mathrm{g}^{\mathrm{M}}$ curves for the binary systems, provided by the new model, have allowed the remarkable improvement of the fitting.

\subsection{Application of the new model to the type 3 system}

Correlation of the type 3 system, previously correlated with NRTL, was repeated with the modified NRTL model (Eq. (1)). Initial values for the $F_{i}$ and $A_{i j}$ parameters were proposed using the best set of parameters obtained with NRTL, and qualitatively searching for new sets of parameter values that simultaneously reproduced equilibrium tangent points close to the experimental ones for the binary $g^{\mathrm{M}}$ curves and the cross-sections $g^{\mathrm{M}}$ curves along the experimental tie-triangle sides. Parameters and deviations for NRTL and the modified model are collected in Table 2 where standard deviations (Eq. (3)) are also shown.

Fig. 11 shows the comparison between experimental data and correlation results for the new model in a composition diagram, together with the binary $g^{\mathrm{M}}$ curves. It can be observed that for the calculated tie-triangle to approach the experimental one while using NRTL, it is required that the LLE calculated compositions for the 1-2 binary subsystem be much closer to one another and, therefore, very far from the experimental ones. However, the new model overcomes this deficiency and considerably improves the correlation of this system, compared with Fig. 5, since it can reproduce a narrow tie-triangle together with a more open 1-2 binary subsystem.

Fig. 12 shows the $3 \mathrm{D} g^{\mathrm{M}}$ surface for type 3 system with the proposed model. The $g^{\mathrm{M}}$ binary curves are considerably different from the curves in the modified model, compared with results obtained with NRTL (Fig. 6). Curvature for 1-3 $g^{\mathrm{M}}$ binary is more marked compared with the other binaries, and the tangent line in this binary has a higher slope than the one described with NRTL model. The curvature of $g^{\mathrm{M}}$ function for 1-2 binary is a little more pronounced than NRTL and $g^{\mathrm{M}}$ function for 2-3 binary has a similar curvature with somewhat higher $g^{\mathrm{M}}$ values. Both binaries 1-2 and 2-3 keep tangent lines with slopes similar to NRTL model results. As a consequence of these differences with the modified model, tie-line slopes of the LLE region starting at 1-3 binary are increased while tie-line slopes starting from the other binaries keep with a similar slope. Besides, higher curvatures of binaries 1-2 and 1-3 lead to greater LLE regions starting from the corresponding binaries. All these characteristics help in predicting a smaller tie-triangle closer to the experimental one.

\section{Conclusions}

Summarizing, we can conclude that the high deviations in correlation results provided by NRTL (as representative of local composition models), and the existence of gaps in the predicted behaviour of the $g^{\mathrm{M}}$ vs. composition curves for the binaries, show that more flexible equations are needed.

The topological analysis of the $g^{\mathrm{M}}$ function is a powerful tool that must be properly used to guarantee the coherency among the calculated equilibrium and the experimental data. The knowledge of the geometrical conditions that the surface has to fulfil to be coherent with a given equilibrium, may help in the development of both empirical and theoretical models to reproduce the phase equilibria with the accuracy required in design calculations.

A simple modification based on the NRTL model is proposed as an example, which takes into account the topology required for the $g^{\mathrm{M}}$ function, shows a great flexibility, fills the gaps of NRTL in the $\mathrm{g}^{\mathrm{M}}$ vs. composition curves and improves the correlation results for the selected examples shown.

\section{List of symbols}

$A_{i j} \quad$ binary interaction parameters (K)

$\alpha_{i j} \quad$ non-randomness NRTL factor

$a_{i, s}^{k} \quad$ activity of component i of tie-line $s$ in phase $k$

$G^{E} \quad$ excess Gibbs energy $\left(\mathrm{J} \mathrm{mol}^{-1}\right)$

c number of components

$F_{i} \quad$ new model parameters (dimensionless)

$G^{\mathrm{M}}, g^{\mathrm{M}}$ Gibbs energy of mixing ( $\mathrm{J} \mathrm{mol}^{-1}$ and dimensionless, respectively)

$g_{\min }^{\mathrm{M}} \quad$ global minimum Gibbs energy of mixing (dimensionless)

LLE liquid-liquid equilibrium

LLLE liquid-liquid-liquid equilibrium

LSE liquid-solid equilibrium

LLSE liquid-liquid-solid equilibrium

LLShE liquid-liquid-hydrated solid equilibrium

LShE liquid-hydrated solid equilibrium

$n \quad$ tie-line number

$P \quad$ number of phases

$R \quad$ gas constant $\left(\mathrm{J} \mathrm{K}^{-1} \mathrm{~mol}^{-1}\right)$

$T \quad$ temperature (K)

$x_{i, \text { min }} \quad$ molar fraction of component $i$ in the minimum of the Gibbs energy of mixing

\section{Superscripts}

exp experimental value

cal calculated value

\section{Subscripts}

$h, i, j \quad$ Components $h, i, j$ 
$s \quad$ tie-line $s$

$k \quad$ phase $k$

\section{Acknowledgement}

The authors gratefully acknowledge financial support from the Vice-presidency of Research (University of Alicante) and Generalitat Valenciana (GV/2007/125).

\section{Appendix A. Supplementary data}

Supplementary data associated with this article can be found, in the online version, at doi:10.1016/j.fluid.2009.12.026.

\section{References}

[1] J.M. Sørensen, Correlation of Liquid-Liquid Equilibrium Data, Thesis, Instituttet for Kemiteknik Danmarks Tekniske Højskole, Denmark, 1980.

[2] M.C. Annesini, F. Gironi, L. Marrelli, J. Chem. Eng. Data 39 (1994) 502-505.

[3] N. Barnes, M. Gramajo de Doz, H.N. Sólimo, Fluid Phase Equilib. 168 (2000) 217-227.

[4] F.S. Santos, S.G. d’Ávila, M. Aznar, Fluid Phase Equilib. 187-188 (2001) 265274.

[5] B.C. Roy, M.R. Awual, M. Goto, J. Appl. Sci. 7 (2007) 1053-1060.

[6] G.R. Vakili-Nezhaad, M. Mohsen-Nia, V. Taghikhani, M. Behpoor, M. Aghahosseini, J. Chem. Thermodyn. 36 (2004) 341-348.

[7] M.A.P. Pereira, M. Aznar, J. Chem. Thermodyn. 38 (2006) 672-677.

[8] J.M. Sørensen, W. Arlt, Liquid-Liquid Equilibrium Data Collection, Chemistry Data Series, vol. V, Part 2, DECHEMA, 1980.

[9] DECHEMA Data Preparation Package (DPP) DECHEMA e.V.

[10] ChemCAD 5.1.5, 2000, Chemstations Inc.

[11] R.E. Treybal, Extracción en fase líquida, first ed., UTEHA, 1968.

[12] F. Becker, P. Richter, Fluid Phase Equilib. 49 (1989) 157-166.

[13] D. Patterson, Polym. Eng. Sci. 22 (1982) 64-73.
[14] R. Stateva, P. Cholakov, S. Georgi, A.A. Galushko, W.A. Wakeham, Chem. Eng. Sci. 55 (2000) 2121-2129.

[15] M. Kahlweit, R. Strey, G. Busse, J. Phys. Chem. 94 (1990) 3881-3894

[16] J.G. Andersen, N. Koak, T.W. de Loos, Fluid Phase Equilib. 163 (1999) 259-273.

[17] Z. Huan, G.H. Van Bochove, T.W. de Loos, Thermodynamics 49 (2003) 745-752.

[18] V. Boddu, A. Krishnaiah, D.S. Viswanath, J. Chem. Eng. Data 46 (2001) $1172-1175$

[19] V.B. Veljković, M.L. Lazić, M.Z. Stanković, World J. Microb. Biot. 22 (2006) 519-523.

[20] D. Dionysiou, M. Tsianou, G. Botsaris, Ind. Eng. Chem. Res. 39(2000)4192-4202.

[21] A. Marcilla, M.M. Olaya, M.D. Serrano, J. Chem. Eng. Data 52 (2007) 2538-2541.

[22] M.M. Olaya, J.A. Reyes-Labarta, R. Velasco, I. Ibarra, A. Marcilla, Fluid Phase Equilib. 265 (2008) 184-191.

[23] J.A. Reyes-Labarta, M.M. Olaya, R. Velasco, M.D. Serrano, A. Marcilla, Fluid Phase Equilib. 278 (2009) 9-14.

[24] A. Marcilla, M.M. Olaya, M.D. Serrano, R. Velasco, J.A. Reyes-Labarta, Fluid Phase Equilib. 281 (2009) 87-95

[25] M.M. Olaya, A. Marcilla, M.D. Serrano, A. Botella, J.A. Reyes-Labarta, Ind. Eng. Chem. Res. 46 (2007) 7030-7037.

[26] A. Marcilla, J.A. Reyes-Labarta, M.M. Olaya, M.D. Serrano, Ind. Eng. Chem. Res. 47 (2008) 2100-2108.

[27] H. Renon, J.M. Prausnitz, AIChE J. 14 (1968) 135-144.

[28] C.-C. Chen, H.I. Britt, J.F. Boston, L.B. Evans, AIChE J. 28 (1982) 588-596.

[29] C.-C. Chen, L.B. Evans, AIChE J. 32 (1986) 1655-1664.

[30] G. van Bochove, G.J.P. Krooshof, T.W. de Loos, Fluid Phase Equilib. 171 (2000) 45-58.

[31] M.L. Michelsen, The isothermal flash problem. Part I. Stability, Fluid Phase Equilib. 9 (1) (1982) 1-19.

[32] S.W. Wasylkiewicz, L.S. Sridhar, M.F. Doherty, M.F. Malone, Ind. Eng. Chem. Res 35 (1996) 1395-1408.

[33] M.M. Olaya, J.A. Reyes-Labarta, I. Ibarra, M.D. Serrano, A. Marcilla, Study of the relations between the binary mixture Gibbs energy curves and the calculated ternary LL equilibrium using NRTL model, in: VII IberoAmerican Conference on Phase Equilibria and Fluid Properties for Process Design, Equifase 2006, Morelia, Michoacán, México, October 21-25, 2006.

[34] M.M. Olaya, I. Ibarra, J.A. Reyes-Labarta, M.D. Serrano, A. Marcilla, Chem. Eng. Educ. 41 (2007) 218-224.

[35] G. Iglesias-Silva, A. Bonilla-Petriciolet, P.T. Eubank, J.C. Holste, K.R. Hall, Fluid Phase Equilib. 210 (2003) 229-245. 